\title{
Poder estructural agroalimentario en países emergentes. Algunas limitantes estructurales de la integración y cooperación internacional en materia agroalimentaria*
}

por Juan José Borrell**

\section{- Resumen}

El artículo formula un breve análisis del posicionamiento internacional de los países emergentes del Cono Sur, en particular de Argentina, a partir de las relaciones de integración y cooperación económica en materia agroalimentaria. Para tal fin se desarrolla el concepto de poder estructural agroalimentario en base a la noción de poder estructural de Susan Strange. Dicho enfoque a diferencia de la visión propuesta por el institucionalismo liberal de régimen alimentario, permite comprender la dinámica del sistema agroalimentario internacional en su real dimensión. El supuesto crítico que se plantea es que si bien la explotación de materias primas agroalimentarias ha generado coyunturalmente ganancias para las economías emergentes, el poder estructural agroalimentario a partir de relaciones de integración y cooperación de tipo asimétrico implica un posicionamiento periférico y dependiente en la economía global, viniendo en consecuencia a reforzar la configuración del poder estructural internacional.

\section{- Palabras Clave}

poder estructural agroalimentario - sistema agroalimentario - régimen alimentario - países emergentes

\section{Inglés | English}

\section{Agrifood structural power in emerging countries. Some structural limitations of international integration and cooperation regarding agrifood}

\section{- Abstract}

The article makes a brief analysis of the international positioning of the emerging countries of South America, particularly Argentina, from the relations of economic integration and cooperation regarding agrifood. To this end the concept of agrifood structural power is developed based on the notion of structural power of Susan Strange. This approach unlike the vision proposed by the liberal institutionalism of food regime enables to understand the dynamics of the international food system in its real dimension. The critical postulation at issue is that while the exploitation of agrifood commodities has generated conjunctural profits for emerging economies, the agrifood structural power due to asymmetric relations of integration and cooperation implies a peripheral and dependent position within global economy, thus coming to reinforce the configuration of the international structural power.

\section{- Key words}

Agrifood structural power - agrifood system - food regime emerging countries

\footnotetext{
* Una versión anterior de este artículo se elaboró para un seminario de Economía Política Internacional a cargo de Eiiti Sato y José Fernández Alonso en el Doctorado en Relaciones Internacionales de la Universidad Nacional de Rosario. Me resultaron muy valiosas las sugerencias y comentarios que para aquella versión me hicieron María Elena Lorenzini y Nicolás Creus. Agradezco también las sugerencias efectuadas por el árbitro anónimo. Huelga aclarar que los errores y omisiones de este trabajo corren por cuenta del autor.

** Magister en Estrategia y Geopolítica (IUE-ESG). Docente en la Universidad Nacional de Rosario y en la Escuela Superior de Guerra (IUE, Buenos Aires). Ha integrado la Delegación Oficial Argentina ante el CFS del Organismo de Naciones Unidas para la Alimentación y la Agricultura (FAO), Roma, 2011-2015. Correo electrónico: juanjoseborrell@gmail.com
} 
«Food is a weapon»

Earl Butz, 1974

(Secretario de Agricultura de Estados Unidos, 1971-76) del poder estructural internacional a partir de relaciones de integración y cooperación de tipo asimétrico.

\section{"U.S. needs to lead in feeding the world» Richard Lugar 2014 \\ (Senador Estados Unidos, 1977-2013)}

\section{Introducción}

El presente artículo formula un breve análisis del posicionamiento internacional de los países emergentes del Cono Sur, en particular de Argentina, a partir de la explotación de materias primas agroalimentarias.

Está dividido en dos partes: la primera sección plantea la existencia de un poder estructural agroalimentario, en base a la conceptualización de poder estructural de Susan Strange y de las posibles estructuras de poder secundarias de la economía política internacional (Strange, 1994). El desarrollo de dicho concepto, el cual se diferencia teóricamente de la noción de régimen alimentario del institucionalismo liberal (Hopkins y Puchala 1978), se realiza con el objetivo de comprender con mayor precisión y realismo la dinámica del sistema agroalimentario internacional y de este modo comprender las dinámicas agroalimentarias locales.

La segunda sección indaga brevemente a partir de los desarrollos recientes en el Cono Sur en materia de explotación de materias primas agroalimentarias, si tales dinámicas en los países emergentes responden a un fenómeno de difusión del poder o bien tienden a reafirmar las estructuras de poder existentes. De aquí que las tendencias agroalimentarias a nivel local comprendidas en el marco del poder estructural agroalimentario permiten pensar una respuesta al interrogante central sobre el posicionamiento económico internacional de Argentina.

En este sentido, para el interrogante de base ¿qué incidencia económico-política internacional tiene la explotación de materias primas agroalimentarias de Argentina?, el supuesto crítico que se plantea es que si bien la explotación de materias primas ha generado coyunturalmente ganancias para las economías emergentes de la región reflejándose incluso en los flujos globales de comercio y finanzas, el poder estructural agroalimentario permite un posicionamiento periférico y dependiente en la economía global, viniendo en consecuencia a reforzar la configuración

\section{Entre el régimen alimentario global y el poder estructural agroalimentario}

Los principios básicos del sistema agroalimentario internacional se analizaron y pusieron en discusión por primera vez en espacios académicos a la luz del concepto de régimen.

El cimbronazo que sufrió el entero sistema agroalimentario internacional tras la crisis del precio de los alimentos de 1973-74, a caballo de otros procesos desencadenados como la quiebra del sistema cambiario de Bretton Woods y la devaluación del dólar, la suba del precio del petróleo y el agotamiento de las reservas granarias internacionales, contribuyó a que el interrogante formulado al interior de organizaciones multilaterales como el Organismo de Naciones Unidas para la Alimentación y la Agricultura (FAO) sobre la continuidad a futuro de los pilares del sistema agroalimentario establecido en la posguerra se abordaran también teóricamente. En el campo académico norteamericano de las relaciones internacionales a mediados de los 70s y bajo el paraguas de las reformas de orden económico tras los procesos antes mencionados, surgió un reto de cuño liberal a la visión realista estructural vigente focalizado desde el concepto de régimen internacional $^{1}$. Específicamente para el caso del sistema alimentario global, Raymond Hopkins y Donald Puchala tras la crisis alimentaria de 1973-74 buscan sistematizar un análisis sobre la lógica del mismo y editan en 1978 un volumen que primero se publica como tema especial en International Organization (Vol. 32, № 3) y luego por la Universidad de Wisconsin (Hopkins y Puchala, 1978), donde entre las colaboraciones de distintos especialistas sobre la economía política global de los alimentos ellos desarrollan el concepto de régimen alimentario global. El capítulo en el que lo presentan, "Perspectives on the International Relations of food" se reedita puntualmente más tarde como "International Regimes: lessons from inductive analysis" para International Organization (Vol 36, No 2) en 1982, para finalmente publicarse en el libro editado por Stephen Krasner sobre regímenes internacionales dentro de la sección "Perspectivas Grotianas" (Krasner, 1983).

Aunque los autores asentaron los basamentos conceptuales para futuras discusiones y análisis sobre las dinámi- 
cas agroalimentarias locales e internacionales, su visión institucionalista liberal no difería sustancialmente del esquema que es moneda corriente en el seno de organismos multilaterales y que resulta insuficiente para dar real cuenta de procesos estructurales.

En principio, tal como caracterizan Hopkins y Puchala, el sistema alimentario global refiere a las funciones interconectadas de producción, distribución y consumo, y a los medios de interconexión a través de transacciones públicas y privadas. El sistema alimentario global estructuralmente está compuesto de centros de producción, centros de consumo y canales de distribución e intercambio, comerciales y concesionales. Para ellos las condiciones que prevalecen en el sistema alimentario global no ocurren azarosamente ni enteramente en respuesta a imperativos agrícolas o económicos; suceden porque hay personas que toman decisiones sobre la producción, distribución y consumo que, de acuerdo con normas comúnmente aceptadas y ampliamente prevalecientes, le otorgan legitimidad a ciertas prácticas y declaran ilegítimas otras. Los conjuntos de tales normas, guías prevalecientes en determinados momentos, constituyen regímenes. De aquí que un régimen es un conjunto de reglas, normas o expectativas institucionales formales e informales que rigen un sistema social; donde regir significa controlar, regular o bien brindar orden, continuidad o predictibilidad a partir de influir en las prácticas que dan forma al comportamiento general de las diversas partes del sistema alimentario (Hopkins y Puchala, 1978).

Si bien el sistema alimentario global aparece descentralizado en subsistemas nacionales donde la mayor parte de la producción, consumo e intercambio tiene lugar, está profundamente afectado por las transacciones del mercado internacional, y en particular ha sido "estadosunidos-céntrico" desde la posguerra, es decir basado principalmente en la economía política doméstica de Estados Unidos. En este sentido las reglas del régimen se originan como políticas nacionales norteamericanas que se negocian y coordinan a nivel internacional, por propósito o por defecto, por acuerdo multilateral o dictado unilateral.

Según el abordaje conceptual específico de régimen de Hopkins y Puchala, las normas y principios que guían y restringen las relaciones internacionales de los alimentos desde los últimos años de la década del 40 hasta principios de los 80s (como el respeto por el libre mercado, la soberanía nacional, la libre circulación de información científica, y el evitar las hambrunas), surgen de la política descentralizada mundial y de las interacciones de los esta- dos y otras organizaciones de acuerdo a un entendimiento compartido o intersubjetividad según las preferencias de los actores. Aunque las capacidades de negociación tiendan a ser asimétricas, serían en su mayor parte reglas voluntariamente concertadas.

Para los autores el régimen alimentario a principios de los 70s sacó a la luz sus defectos para terminar sufriendo en los 80s algunos cambios de tipo marginal. La falta de acuerdo internacional desde la Conferencia Mundial de Alimentos de 1974 y las sucesivas cumbres respecto a los stocks de reserva granaria fue interpretada como una reducción de los recursos disponibles para reforzar las normas del régimen apuntaladas por el poder norteamericano, fruto de una cuestión mayor, según los autores, que era la percepción del declive de la hegemonía de Estados Unidos en el orden internacional.

Contemporáneamente a los autores mencionados y en el mismo volumen editado por Krasner, Susan Strange (1982) realiza una minuciosa crítica del concepto de régimen internacional la cual, llevada a la noción particular de régimen alimentario, se ajusta más a comprender la dinámica del sistema agroalimentario mundial tanto en el contexto de los 80 s como en la actualidad, desde una perspectiva no idealizada de la economía política internacional.

En primer lugar, Strange no concibe en los 80 s ni en los 90s que, en el orden internacional, Estados Unidos haya sufrido un declive hegemónico. En lo único que existe una disminución en los 80 s es en la voluntad de intervenir en los mecanismos del mercado mundial, pero en las estructuras globales de seguridad, del sistema de crédito, del sistema de bienestar y de los sistemas de conocimiento y comunicaciones, no existe indicio fáctico de una merma del poder norteamericano (Strange, 1982; Strange, 1994). En particular, el peso, capacidad de influencia y proyección de las corporaciones del agribusiness de ese país, secretarías de gobierno, agencias de desarrollo internacional como USAID, grupos privados de productores y lobbys de presión en materia agroalimentaria, tampoco mermaron. De hecho, la creación de la Organización Mundial del Comercio (OMC) en 1995 tras la Ronda Uruguay del GATT y las álgidas negociaciones para liberalizar los acuerdos de comercio agroalimentario internacional, se dio en gran parte por la presión del agribusiness norteamericano en un contexto de mega-expansión global tras el fin de la Guerra Fría, período que precisamente es considerado como de reafirmación hegemónica y unipolaridad ${ }^{2}$.

2. Para una visión en perspectiva sobre el debate de la declinación norteamericana ver el capítulo 6, "Power transition: the question of American decline", en NYE, Joseph (2011), The future of power, Public Affairs, Nueva York. 
En segundo lugar, la visión de Hopkins y Puchala, como la de la mayoría de los politólogos, dice Strange, peca de estadocéntrica. Existe para ella un vicio epistemológico en los subcampos académicos de las Ciencias Políticas de pasar todos los fenómenos por el tamiz conceptual del actor racional Estado. Según Strange, los Estados si bien son unos de los actores principales del sistema internacional no son entidades monolíticas, por lo que no poseen un criterio unívoco de acción; pueden estar restringidos en su acción política exterior ya que la misma no es totalmente independiente del plano doméstico, ni de otras esferas, como las finanzas globales. Por otro lado, el empleo del concepto de régimen, el cual pertenece a un esquema heredado de los estudios de gobierno y del derecho internacional, encorseta el análisis de lo mundial al no ponderar factores dinámicos como la economía política internacional y el cambio tecnológico, aspectos clave para comprender la lógica particular del sistema agroalimentario. Además, la visión del régimen vigente es norteamericana, tendiente a sostener como patrón de principios y reglas las del statu quo, en el sentido de que los análisis dan por supuesto que a nivel internacional voluntariamente "todos quieren más y mejores regímenes" como el existente y que por caso, las preocupaciones de los países del bloque socialista en los $80 \mathrm{~s}$ como las del mundo en desarrollo, no pasarían por lograr mayor autonomía, poder o ventajas de intercambio y riquezas sino por compartir la meta "colectiva mundial" de conservar, validar y aceptar irremediablemente los principios del régimen internacional vigente (Strange, 1982).

En este sentido la miopía de Hopkins y Puchala se origina al forzar la transferencia del concepto de régimen (equivalente para ellos al de sistema internacional) al juego sumamente dinámico y azaroso del mercado agroalimentario, y a la vez por sobrevalorar la capacidad de influencia de organismos multilaterales como FAO en los criterios de conducta exterior de los estados y en el establecimiento de prioridades en las agendas nacionales de los gobiernos a negociar con sus actores domésticos. Desde una óptica opuesta, resulta irreal encorsetar un sistema como el agroalimentario a un ramillete de principios ideales cuando precisamente la lógica que opera es la de "hablar de libre mercado pero practicar el mercantilismo" y, por otro lado, también pensar las negociaciones multilaterales de forma horizontal por concebir a los organismos internacionales en las que tienen lugar como una suerte de reducto del sacrosanto consensus gentium grotiano. No casualmente afirman como una generalización conclusiva que "la efectividad en términos de conformidad con las reglas y procedimientos de cualquier régimen depende ampliamente del consenso o de la aquiescencia de los participantes" (Hopkins y Puchala, 1982:273).
Derivada de lo anterior es la atribución de una sobredimensionada convocatoria moral sobre los estados por parte de los organismos multilaterales con temas como la inseguridad alimentaria mundial y la ampliación de un libre mercado internacional. Más allá de la promesa humanitarista y liberal de los debates coyunturales en las cumbres de FAO en contextos de crisis alimentaria, una cara de la verdad es que la ayuda alimentaria de posguerra en gran parte realizada por Estados Unidos respondía a la política gubernamental de apoyar con subsidios la producción agrícola doméstica y la consecuente necesidad de dar curso al excedente producido en mercados externos bajo la forma de ayuda humanitaria o ventas concesionales para que no entraran en los circuitos comerciales y deprimieran los precios (Friedmann, 1993). Esta práctica contribuía a generar dependencia de las importaciones de alimentos en los países del llamado Tercer Mundo al desincentivar las producciones locales socavando la autonomía alimentaria, ya que no era rentable competir con importaciones subsidiadas. La misma lógica, también en un marco ideal de libre mercado total de cereales, hubiese barrido con las producciones locales de los países europeos de no haber aplicado sus gobiernos medidas proteccionistas y barreras arancelarias desde la posguerra hasta la actualidad.

La otra cara de la dinámica respondía a criterios estratégicos de seguridad nacional y a concebir la ayuda alimentaria, a partir del año 1947 con la doctrina Truman y sus versiones posteriores como la Public Law 480 de 1954 y el programa Food for Peace luego World Food Programme (WFP) de los 60s, como un instrumento de la contención a la expansión mundial del comunismo. Donde había hambre endémico existía el fermento del descontento social y la revuelta política, caldo de cultivo propicio para un aprovechamiento soviético para expandirse en el Tercer Mundo. De aquí que tanto la "ayuda alimentaria" como la transferencia tecnológica de la matriz agroindustrial del conglomerado del agribusiness norteamericano, ideado en el seno de la Fundación Rockefeller, sirvieron como recursos de política exterior en el contexto de la Guerra Fría (Perkins, 1997).

Ambas prácticas se resignificaron a partir de las mega-ventas de cereal a la URSS por los grandes traders cerealeros norteamericanos a principios de los 70s. Dichas ventas, las cuales, al principio, fueron secretas y a espaldas del Congreso de Estados Unidos, implicaron sólo para la cosecha 1972-73 una compra por parte de la URSS de casi las tres cuartas partes de todo el cereal comerciado a nivel mundial en el período (Friedmann, 1993). Aunque sirvió como moneda de cambio entre las dos superpotencias en el contexto de la Détente, la maniobra contribuyó a disparar los precios del cereal y por lo tanto a disminuir sustancialmente las reservas para ayuda humanitaria. 
En síntesis, los referentes del institucionalismo liberal no dan suficiente relieve a los juegos geoestratégicos de poder y seguridad del sistema internacional (Grieco, 1988), que también engloban al sistema agroalimentario mundial. No ponderan que uno de los factores decisivos del aparente cambio de régimen alimentario a mediados de los 70s, por la merma de reservas y la disparada de precios, fue la integración del bloque soviético al circuito comercial mundial de cereales, primero como un negocio privado de Cargill y Continental, luego como instrumento diplomático de la Administración Nixon. Es decir, un giro radical en las reglas del régimen de posguerra cuyo leitmotiv había radicado en mantener el máximo orden económico al interior de la alianza occidental. Tampoco sopesan, entre los factores de su análisis, el impacto estructural en la economía política y financiera internacional de la crisis del sistema de Bretton Woods y el brusco giro de la política monetaria norteamericana. Finalmente, no terminan distinguiendo entre un uso declamativo de principios liberales universalistas (el libre mercado, el flagelo del hambre, la libre circulación de información científica y la inviolabilidad de las soberanías nacionales) de las dinámicas concretas de poder y competencia de los diversos actores públicos y privados involucrados en el sistema agroalimentario mundial. Por ello, el supuesto régimen alimentario no modificó el contexto en que los estados toman decisiones en materia agroalimentaria sino que fueron las decisiones por razones de seguridad estratégica de los actores más poderosos del tablero internacional y las fuerzas económicas estructurales las que cambiaron la esencia del régimen.

Una perspectiva realista clásica con un enfoque en el poder conductual y en la posesión de recursos naturales para la medición del poder nacional y el ejercicio de acciones coactivas, como teorizaban Hans Morgenthau y Klaus Knorr, tampoco serviría a los efectos de comprender la dinámica compleja de los procesos de integración y cooperación del sistema agroalimentario internacional. Un sistema casi totalmente articulado por corporaciones privadas mientras es potenciado por el sector estatal para la obtención de beneficios mutuos, opera sinérgicamente como en los casos de la ayuda alimentaria y la difusión de la matriz agroindustrial en el Tercer Mundo; pero ¿hasta qué punto el poder estatal puede disponer del agribusiness como un instrumento de política exterior? Un sector, durante los debates que se suscitaron con el bloqueo de la Organización de Países Exportadores de Petróleo (OPEC) en 1973-74 y la situación de vulnerabilidad que generó en
Estados Unidos la interrupción del suministro de petróleo, consideraba implementar contramedidas de guerra económica contra los países objetivo empleando como instrumento las exportaciones agrícolas. Sin embargo, diversos especialistas consideraban, tanto desde miradas realistas como liberales (Schneider, 1976; Christensen, 1977; Rothschild 1976), que el rango de opciones que tenía Estados Unidos para ejercer Food Power era muy estrecho y hasta contraproducente, ya que "el impacto de retener exportaciones agrícolas eventualmente servirá de estímulo para que la nación objetivo busque fuentes alternativas de suministro exterior o bien promueva la producción doméstica" (Schneider, 1976:56). Por caso, los embargos, por parte del gobierno norteamericano, de soja a Japón en 1973 y de trigo y maíz a la URSS como represalia tras la invasión a Afganistán en 1980 quedaron como lecciones de groseros yerros, y pusieron en evidencia que el gobierno no puede ejercer control sobre la comercialización de cereales, es decir, carece de los recursos políticamente disponibles para instrumentar acciones de poder conductual en base al suministro de alimentos. Las "agencias de comercio y las corporaciones transnacionales y sus subsidiarias eran capaces de usar transacciones complejas y embarques cruzados para organizar el comercio por fuera del conocimiento y mucho menos el control del gobierno norteamericano y en verdad del de cualquier estado" (Friedmann, 1993:41).

Como señala Strange, es la dinámica en las estructuras de poder lo que en verdad determina, en este caso, las relaciones internacionales de los asuntos alimentarios. Sosteniendo hasta una década después, en la reedición de States and Markets, su crítica de los 80s al concepto de régimen internacional busca elucidar la compleja difusión del poder en las estructuras de la economía política internacional proponiendo preguntarse quién obtiene qué cosa, quién se beneficia y quién pierde, quién acarrea los riesgos y quién se los ahorra, quién obtiene las oportunidades y a quién se le niegan, sea de bienes y/o servicios (Strange, $1994)^{3}$.

La autora argumenta que el poder estructural confiere el poder de decidir cómo las cosas deben ser hechas, el poder de dar forma a los marcos dentro de los cuales los estados se relacionan unos con otros, con la gente o con las corporaciones, en tanto resultado de la interacción de la influencia deliberada y la influencia no intencional de las decisiones y de las no decisiones tomadas por gobiernos y

3. Para un abordaje temprano del concepto de poder estructural en base a la síntesis de nociones previas de poder en el nivel estructural diferenciado del nivel conductual y de meta-poder ver CHRISTENSEN, Cheryl (1977), "Structural power and national security", en KNORR, Klaus; TRAGER, Frank (ed.), Economic issues and national security, UP Kansas; Lawrence, págs. 127-159. Para una crítica de diferentes conceptualizaciones de poder estructural ver GUZZINI, Stefano (1993), “Structural power: the limits of neorealist power analysis”, International Organization, Vol. 47, 3,

págs. $443-478$. 
por otros actores. Está compuesto por cuatro estructuras primarias distinguibles pero interrelacionadas, las cuales implican el control de la seguridad, la producción, el crédito, y el conocimiento, las creencias e ideas. Es decir, el poder estructural yace con aquellos en posición de ejercer control sobre la seguridad de las personas (amenazarla o preservarla), yace también con aquellos capaces de decidir y controlar el modo de producción de bienes y servicios para la supervivencia, en aquellos capaces de controlar el suministro y distribución del crédito, y por último en quienes poseen el conocimiento y pueden decidir los términos de acceso al mismo (Strange, 1994).

Estas cuatro estructuras primarias juegan a su vez el gran papel de dar forma a lo que serían estructuras secundarias, identificando también cuatro sistemas (el transnacional de transporte, de comercio, de suministro de energía, y de bienestar y desarrollo internacional), aunque considerando que la elección es arbitraria y que igualmente sería lógico incluir alguna otra estructura secundaria como la "del sistema alimentario mundial, dado que los alimentos para las personas son tan vitales para la producción como la energía para las máquinas" (Strange, 1994:139).

Allanado entonces el camino conceptual por la autora, se puede referir a un poder estructural alimentario en tanto estructura secundaria del poder estructural, el cual está en estrecha interrelación con las estructuras secundarias de transporte, comercio, energía y desarrollo internacional, y el cual a su vez como característica principal comparte con éstas el hecho de que a pesar de ser un marco en el que se toman decisiones está determinado por las estructuras primarias de seguridad, producción, crédito y conocimiento. Este poder estructural alimentario, o mejor agroalimentario, tiene también como característica común con las otras estructuras secundarias que, si bien la autoridad del estado sobre la economía y la sociedad está institucionalizada en ministerios de transporte, de agricultura, de seguridad social y de energía asumiendo en general la responsabilidad de velar por los mismos, existe también una dimensión transnacional de mercados globales y la influencia de otros estados que la autoridad del estado tiene que tener en cuenta (Strange, 1994).

De aquí que surja la pregunta sobre la manera en que el poder estructural ha dado forma a la economía política internacional del sistema agroalimentario y la manera en que distribuye costos y beneficios, riesgos y oportunidades a los diferentes grupos, corporaciones y organizaciones dentro del sistema. Respecto a su relación con las otras estructuras secundarias, ¿quiénes controlan el transporte marítimo de materias primas agroalimentarias? ¿Quiénes son los principales comercializadores agroalimentarios y qué porcentajes del mercado manejan? De lo producido a nivel mundial, ¿qué porcentaje se destina a ayuda humanitaria y qué participación tiene cada país?

Respecto a cómo el poder estructural agroalimentario es determinado por las estructuras primarias, ¿qué papel juega el sistema agroalimentario en las ponderaciones de seguridad estratégica? ¿Cuáles son las principales materias primas producidas, con qué insumos y qué porción corresponde a cada actor? ¿Cómo impactan en las economías emergentes las cotizaciones de las commodities agrícolas de Chicago y el mercado de valores de Nueva York? ¿Quiénes controlan el know-how agrobiotecnológico y monopolizan el sistema de patentes?

Teniendo en vista los interrogantes anteriores, aunque sin espacio para explorar una detallada radiografía del sistema agroalimentario, el aporte conceptual de Strange permite orientar el análisis hacia la problemática del posicionamiento de los países emergentes del Cono Sur según la configuración de los sistemas agroalimentarios locales en relación a las dinámicas de la economía política internacional.

\section{Poder estructural agroalimentario en países emergentes: ¿¿difusión del poder o re- afirmación de la matriz estructural?}

La disparada en 2007-2008 de los precios mundiales de las materias primas agrícolas volvió a situar en foros internacionales de alto nivel y organismos multilaterales la discusión sobre las causas estructurales del fenómeno. La respuesta de gran número de países importadores para blindarse y exportadores para aprovechar la suba de las cotizaciones de cereales retroalimentó la tendencia generando acusaciones informales de distorsión de precios que derivaron en el clásico debate entre los países desarrollados y los en desarrollo exportadores sobre las barreras arancelarias y los subsidios agrícolas.

Si bien la crisis financiera mundial de fines de 2008 y la consecuente caída de las commodities frustró el sueño de la cornucopia interminable del sistema agroalimentario, las cumbres de 2011 del Committee on World Food Security (CFS) de FAO y la de mandatarios del G-20 abordaron la volatilidad del precio de los alimentos como tema especial (HLPE, 2011). La declaración final no vinculante de FAO así como las iniciativas del G-20 propusieron en términos generales un recetario de buenas intenciones en materia agroalimentaria para fortalecer la integración y cooperación internacional: propender a reducir la excesiva volatilidad del precio de las commodities, mejorar la seguridad alimentaria en los países más vulnerables, optimizar los sistemas de información de mercados agrícolas, rever las políticas en biocombustibles como un factor distorsivo de precios, fortalecer el desarrollo rural y la productividad de 
los pequeños productores, la transferencia tecnológica, e incrementar la producción a nivel mundial.

Exceptuando puntos como la propuesta específica del G-20 de fortalecer la regulación sobre el sistema financiero mundial y el debate sobre el impacto de los biocombustibles, todas las demás cuestiones abordadas han sido ejes permanentes de negociación y controversia internacional que se retrotraen cuatro décadas a la crisis del precio de los alimentos de 1973-74 y que trasuntan invariablemente el esquema analítico rígido e insuficiente formulado desde el institucionalismo liberal.

Sin embargo, desde principios de la década de 1990 y en un contexto de renovación de los organismos multilaterales encargados de regular el libre comercio internacional, como por ejemplo con la creación de la OMC en 1995, se ha venido dando una vigorosa evolución del sistema agroalimentario mundial. La caída de la URSS y la significativa incorporación al comercio internacional de los países del bloque socialista, sumó en paralelo el crecimiento exponencial de la producción de materias primas alimenticias de países emergentes como China, India, Brasil y Argentina (Friedmann, 1993). Por su parte, las reformas liberalizantes y privatizaciones en el contexto de las medidas de ajuste del FMI en América Latina contribuyeron a su vez a respaldar y proyectar la acción de actores corporativos del agribusiness transnacional, quienes consecuentemente expandieron aún más la matriz energético-industrial de explotación agrícola en gran escala, herencia de la Revolución Verde.

En este sentido, la mayor participación de países en desarrollo en el sistema agroalimentario mundial (así como en otros rubros económicos de producción de materias primas, manufacturas y finanzas) ha implicado en las últimas décadas una serie de cambios en el orden económico internacional. Diversos analistas internacionales vislumbran incluso un giro hacia una nueva geografía económica del mundo en la que estaría cediendo lugar el predominio económico norteamericano. En particular se considera que las llamadas economías emergentes se han convertido en el motor del crecimiento global y han crecido a un ritmo superior al de las desarrolladas, lo cual implica que ha aumentado significativamente su participación en el PBI mundial, así como en el comercio, la inversión directa y los mercados financieros internacionales (Orgaz et al., 2011). En tanto grandes productores de materias primas (en particular agroalimentarias), y por los volúmenes intercambiados en las últimas casi dos décadas, los países emergentes de primer y segundo orden parecieran disponer una distribución más simétrica del orden económico internacional (Lee et al., 2012).
Una fotografía actualizada de los volúmenes producidos y flujos comercializados de las principales materias primas agrícolas, arroja que Estados Unidos y Canadá actualmente producen alrededor de 443 millones de toneladas de cereales (esto es trigo, maíz, cebada, sorgo, arroz y avena, entre otros), es decir apenas un $18 \%$ de los 2.486 Mton que se producen en el mundo (OECD-FAO, 2014), mientras que en 1979 proveían el 70\% de los cereales del planeta (Hopkins y Puchala, 1982:261). Respecto a las oleaginosas (soja y girasol, entre otras) Estados Unidos en 1977 exportaba el 54,6\% del total mundial (Friedmann, 1993:46), actualmente produce 89,5 Mton y exporta sólo 38 Mton, un 20,6\% y un $8,7 \%$ respectivamente del total mundial de 434 Mton, mientras que países en desarrollo como Brasil y Argentina han venido a ocupar el segundo y tercer lugar en la producción de oleaginosas con un total conjunto de 127 Mton, es decir un 29\% del total global (OECD-FAO, 2014). Por otro lado China, quien a fines de los 70s era considerado un importador pobre de trigo, maíz y oleaginosas en el sistema alimentario mundial, hoy produce 333 Mton de cereales (excluyendo al arroz del cual es el primer productor del mundo), prácticamente autoabasteciéndose de los mismos y a la vez siendo el mayor importador de oleaginosas con 64 Mton, casi un 15\% del total mundial de 434 Mton (OECD-FAO, 2014). Argentina, quien era considerada hasta los 80 s otro actor importante del sistema alimentario internacional, en particular del subsistema triguero, en la actualidad no llega a producir 10 Mton de trigo, un magro $1,4 \%$ del total mundial de 710 Mton (OECD-FAO, 2014), aunque esto se vea compensado por la exponencial expansión de la producción de soja desde mediados de los 90s y los volúmenes de exportación de biodiesel de soja.

Esta mayor participación en la economía agroalimentaria internacional va incluso de la mano de un fortalecimiento de los instrumentos diplomáticos de los países emergentes quienes en organismos y foros internacionales como el G-20, el G-33 o el grupo AMNA 11, conforman coaliciones en pos de modificar las políticas proteccionistas de subvenciones de los países desarrollados defendiendo una liberalización de la agricultura, o bien negociando unilateralmente acuerdos preferenciales con bloques como la Unión Europea por productos determinados como los biocombustibles.

Ahora vale preguntarse, este esquema de geografía económica internacional de producción y comercio de materias primas agrícolas aparentemente más simétrico respecto al esquema de posguerra, ¿implica entonces una difusión mundial del poder? Que el epicentro del sistema agroalimentario mundial haya dado lugar a la emergencia de diversos polos de producción y competencia agroalimentaria ¿tuvo como correlato una modificación de las princi- 
pales reglas del régimen alimentario del institucionalismo liberal? La mayor participación económica y diplomática de los países emergentes más allá de su declamación por un libre comercio agrícola mundial, ¿logró modificar las normas proteccionistas de la Unión Europea y la práctica de subvenciones norteamericana? ¿Representa el grupo BRICS un desafío al poder estructural agroalimentario de matriz norteamericana? ¿Implica esto para Argentina la oportunidad de ganar mayores porciones del mercado e incidir en el sistema internacional a partir del uso del sistema agroalimentario como instrumento de política exterior?

Entre los especialistas que se han mostrado optimistas respecto al rol que pueden jugar en la economía internacional países como los BRICS, muchos estiman que un mayor peso en la economía global si bien debe ir asociado a una madurez institucional acorde, en verdad "ha fraguado un agrupamiento de los países emergentes sistemáticamente importantes en una coalición blanda de países con intereses comunes" (Orgaz et al, 2011:51). Sin embargo, otras visiones son escépticas respecto a los ajustes mutuos y son críticas de un posible contra-consenso internacional con centro en Pekín. En particular, se resalta que el tipo de relación que ha establecido China, el mayor de los emergentes, con países de América del Sur no es precisamente una relación de cooperación simétrica Sur-Sur. A la inversa, pareciera que "el futuro de esos países estará determinado por una nueva relación Norte-Sur, en el estilo de una típica relación centro-periferia, cuya lógica está basada en la especialización estática de la producción de commodities y de recursos naturales extractivos" (Vadell, 2012:425). Si bien China se presentó como una sustanciosa alternativa para los países de la región tras las crisis de los 90s en base a una complementariedad económica dinamizada por la expansión comercial, además del crecimiento mundial y la suba del precio de las materias primas y la energía hasta la crisis financiera de 2008, el patrón de comercio entre China y América del Sur (en particular Argentina en materia agroalimentaria) ha resultado en una re-primarización de las economías, implicando la expansión de la frontera agrícola un caso tipo de neoextractivismo (Gallagher y Porzecanski, 2009).

Esa dinámica coyuntural vino a su vez a agudizar problemas estructurales en el plano doméstico dado que "las eco- nomías con una estructura productiva menos diversificada y una canasta de exportaciones altamente concentrada en este tipo de productos" (Rozenwurcel y Katz, 2012:26), generó en países como Argentina una voraz política fiscal contra sectores productivos atada a una variable externa sumamente volátil como el precio de las commodities agrícolas, condicionando la suerte del edificio económico del país a los vaivenes de los mercados internacionales por un espejismo de suba sin techo ${ }^{4}$.

Vale destacar que la anterior dinámica de integración económica no ha ido en detrimento del conglomerado de grandes actores del agribusiness transnacional sino que, a caballo del mismo, ha propulsado la expansión en gran escala del sistema agroalimentario desde mediados de los 90s. Por un lado la renovada matriz energético-industrial contribuyó con apoyo deliberado del gobierno local a concentrar el control del know-how agroproductivo en un reducido grupo de corporaciones transnacionales ${ }^{5}$. Por otro lado, el cluster comercializador granario transnacional amplió sus volúmenes de intercambio oligopolizando aún más el circuito nacional y regional de venta de materias primas agroalimentarias ${ }^{6}$. Finalmente, corporaciones foráneas procesadoras de materias primas agrícolas ganaron cada vez una mayor presencia en el mercado doméstico de consumo alimentario.

En este sentido Argentina, desde una mirada de geografía económica, ostenta hoy el tercer puesto mundial en producción de soja con 60 Mton de porotos. Sin embargo, más del $90 \%$ del mercado de semillas transgénicas de soja (y también de maíz) está monopolizado por el sistema de patentes de corporaciones transnacionales de origen norteamericano y europeo y subsidiarias locales. De igual modo una gran proporción de los agroquímicos e insumos está repartida entre un reducido grupo de corporaciones norteamericanas (Monsanto, Dow y Dupont) y europeas (Syngenta, Bayer y Basf). Más del 75\% del cereal y las oleaginosas producidas en Argentina es comercializado internacionalmente por un oligopolio cada vez más cerrado de corporaciones procedentes de Estados Unidos (Cargill, Bunge y $A D M$ ), Europa (Dreyfus y Nidera) y por la mixta británica-china Noble. Por su parte en el mercado de alimentos al público argentino, cada vez es mayor la presencia de grandes corporaciones transnacionales de la indus-

4. El declive del ciclo de bonanza en las commodities agrícolas desnudó la vulnerabilidad estructural del circuito. "El campo tiene menos poder de compra hoy que en los años '90", Diario El Cronista Comercial, 15 Septiembre 2015, Buenos Aires.

5. Respecto al origen del actual modelo, ¿cuáles fueron los factores que determinaron la legalización de la soja transgénica RR en 1996 en Argentina? Más aún ¿cuál fue el criterio de eficiencia ponderado para aprobar la Intacta RR2 de Monsanto en 2012 con lo que ello implica en condicionamientos legales, técnicos y económicos?

6. Para información sobre volúmenes producidos y actores comercializadores año 2014 de Argentina ver Informativo Semanal de la Bolsa de Comercio de Rosario, Año XXXIII, No 1724, 11 de Septiembre de 2015. 
tria alimentaria que determinan a nivel macro hábitos de consumo y concentran mayores porcentajes del mercado. Son ejemplo de ello las europeas Unilever, Nestlé, Danone, Carrefour y ABInBev, y las norteamericanas Coca-cola, Pepsico, Kraft Heinz, Mondelez, Walmart y McDonalds, entre otras de menor tamaño ${ }^{7}$.

De aquí que, desagregando los componentes y actores del sistema agroalimentario local, se entiende que no es desde un esquema de mapamundi con divisiones político-territoriales que Argentina como país exporta a China por caso aceite de soja, ni que es la beneficiaria de ese circuito en tanto actor monolítico. Las corporaciones transnacionales y subsidiarias locales que venden los insumos para la producción (semillas y agroquímicos), así como las que compran a los acopios locales y con sus propios medios transoceánicos de transporte le "venden" a su gemela en Extremo Oriente, quien a su vez revende a mayoristas lo transportado -en general subsidiarios suyos- para procesamiento, forraje o consumo humano al menudeo, son componentes determinantes del sistema y constituyen en palabras de Strange el esquema de quién obtiene qué cosa, quién se beneficia y quién pierde, quién acarrea los riesgos y quién se los ahorra, quién obtiene las oportunidades y quién no del poder estructural agroalimentario ${ }^{8}$.

En este sentido, la expansión en las últimas décadas del sistema agroalimentario local en sus ramas de producción, comercialización y consumo ha sido funcional, en una relación directamente proporcional, a los componentes del poder estructural agroalimentario internacional. La participación agroalimentaria argentina (más allá del rol vital que tiene la renta agrícola para la economía del país), por el carácter asimétrico de integración desarrollada, contribuyó a reforzar los componentes estructurales del sistema agroalimentario internacional. Así, la configuración adquirida del sistema agroalimentario local no hace más que afianzar una posición periférica y dependiente en el orden económico internacional, teniendo cada vez un menor margen de acción para actuar autónomamente en el mismo, dada la paulatina vulneración de los propios medios y recursos.

\section{Conclusiones}

El aporte desde el institucionalismo liberal que en su momento realizaron Hopkins y Puchala con el concepto de régimen alimentario sirvió inicialmente para abordar al sistema agroalimentario como tema de las relaciones internacionales y la economía política mundial; sin embargo, dicho enfoque quedó expuesto como insuficiente. De aquí que el concepto de poder estructural de Strange (el cual pondera la incidencia de las estructuras primarias de seguridad, conocimiento y finanzas), empleado específicamente a los efectos de comprender la lógica real del sistema agroalimentario internacional, tenga un valor inestimable.

Por lo expuesto en la primera sección de este artículo, la existencia de un poder estructural agroalimentario internacional, y en la segunda sección, el tipo de integración económica que Argentina viene teniendo a partir de la explotación de materias primas agrícolas, se da cuenta del supuesto planteado: el poder estructural agroalimentario permite un posicionamiento periférico y dependiente en la economía global, viniendo en consecuencia a reforzar la configuración del poder estructural internacional.

Frente a las renovadas narrativas de cuño humanitario y liberal de los organismos multilaterales queda claro que es el poder estructural agroalimentario el que en verdad articula las relaciones de integración y cooperación del sistema agroalimentario internacional. El cómo producir, con qué tecnología e insumos, cómo vender, a quién y a qué precios, estructura el marco dentro del cual se dan fenómenos a nivel micro de cifras de cosecha y flujos comerciales de cereales. Por lo que, el dato crudo del aumento de la producción de una materia prima agrícola en un país en desarrollo como Argentina o de los términos de intercambio en una coyuntura favorable particular no se traduce en una modificación del poder estructural agroalimentario, menos aún en una modificación del orden económico internacional.

Respecto a las relaciones al interior del grupo de países en desarrollo, que China, Brasil o Argentina puedan catalogarse como emergentes en un rango muy generoso de in-

7. ¿Hasta qué punto se puede referir a una división internacional corporativa en países? La composición financiera de los mayores actores transnacionales agroalimentarios (Monsanto, Bunge, Bayer o Syngenta) indica que los mismos grupos de accionistas y fondos de inversión cruzan transversalmente la propiedad del capital y acciones de las corporaciones, los cuales en su mayoría están radicados en Estados Unidos, por ejemplo Vanguard Group, FMR Llc, Blackrock Fund Advisors o Jennison Assoc. ¿Cómo incide esto estructuralmente en la toma de decisiones y en posibles acciones oligopólicas globales?

8. Grandes corporaciones de origen argentino como Aceitera General Deheza (AGD), Molinos Río de la Plata y Arcor compiten por porciones del mercado del sistema agroalimentario tanto a nivel local como internacional. Aunque su presencia es significativa en el plano doméstico no deja de ser minoritaria respecto a las corporaciones extranjeras, y estar sujeta a la lógica del poder estructural agroalimentario internacional (para no mencionar a los actores locales medianos y pequeños). 


\section{Poder estructural agroalimentario en países emergentes. Algunas limitantes estructurales de la integración y cooperación internacional en materia agroalimentaria}

clusión, no implica tampoco solidaridad automática o cooperación de carácter simétrico. Hay posicionamientos de acuerdo al poder relativo de cada país, y en particular el patrón que se viene desarrollando en América del Sur en materia agroalimentaria respecto al gran emergente chino, que tienden hacia una renovada relación de centro-periferia.

Por el contrario, una menor participación proporcional de Estados Unidos en el circuito agroalimentario internacional tampoco puede leerse como síntoma de declinación hegemónica. Aquellos que incurren, parafraseando a Strange, en el "mito persistente" de la declinación norteamericana ante el crecimiento económico de países en desarrollo señalando una evidente difusión global del poder, pierden de vista, por un lado la ausencia de voluntad política (y de capacidades relativas) de los mayores emergentes de cambiar las reglas de juego estratégico del sistema internacional; y por otro lado, respecto a lo agroalimentario en particular, el hecho indiscutible que es dentro de los límites del poder estructural y gracias a él que los emergentes han podido integrarse y crecer económicamente en el sistema agroalimentario. Lo que ciertamente se ha esparcido por el mundo desde mediados de la década del 40 hasta la actualidad es la matriz agroindustrial conformada por el conglomerado del agribusiness norteamericano en competencia con corporaciones europeas. En otras palabras, la difusión global fue del acceso al uso de los medios de producción, comercialización y consumo agroalimentario, no de la propiedad y control de la matriz estructural.

Aún así, transcurridas más de dos décadas desde el fin de la Guerra Fría y la anunciada retreat of the state según Strange, ante las recientes tendencias en la economía global y el avance de los países emergentes, habría que analizar más en profundidad de qué manera la creciente presencia y reafirmación del control estatal sobre los circuitos económicos internacionales actuales (tanto en el mundo desarrollado como en desarrollo) puede llegar a imprimirle a la lógica de competencia económica un componente de seguridad nacional, y por lo tanto de pugna internacional. 


\section{Poder estructural agroalimentario en países emergentes. Algunas limitantes estructurales de la integración y cooperación internacional en materia agroalimentaria}

\section{Bibliografía}

CHRISTENSEN, Cheryl (1977), "Structural power and national security", en KNORR, Klaus; TRAGER, Frank (ed.), Economic issues and national security, UP Kansas, Lawrence, pp. 127-159

FRIEDMANN, Harriet (1993), "The political economy of food: a global crisis", New Left Review, No 197, pp. 29-57

GALLAGHER, Kevin; PORZECANSKI, Roberto (2009), "China and the Latin America commodities boom: a critical assessment", Political Economy Research Institute, № 192; University of Massachusetts, Amherst

GRIECO, Joseph (1988), "Anarchy and the limits of cooperation: a realist critique of the newest liberal institutionalism", International Organization, Vol. 42, 3, pp. 485-507

HLPE (2011), Volatilidad de los precios y seguridad alimentaria, FAO, Roma

HOPKINS, Raymond; PUCHALA, Donald (ed.) (1978), The global political economy of food, University of Wisconsin, Madison

HOPKINS, Raymond; PUCHALA, Donald (1982), "International regimes: lessons from inductive analysis”, en KRASNER, Stephen (ed.) (1983), International regimes, Cornell University, Ithaca, pp. 61-92

KRASNER, Stephen (ed.) (1983), International regimes, Cornell University, Ithaca

LEE, Bernice et al. (2012), Resources futures, Royal Institute of International Affairs, Londres

OECD-FAO (2014), OECD-FAO Agricultural Outlook 2014-2023, OECD, Paris

ORGAZ, Luis, et al. (2011), "El creciente peso de las economías emergentes en la economía y gobernanza mundiales”, Documentos ocasionales, № 1101, Banco de España, Madrid

PERKINS, John (1997), Geopolitics and the Green Revolution. Wheat, genes, and the Cold War, Oxford University, Nueva York

ROTHSCHILD, Emma (1976), “Food politics”, Foreign Affairs, pp. 285-307

ROZENWURCEL, Guillermo; KATZ, Sebastián (2012), "La economía política de los recursos naturales en América del sur”, en Revista Integración \& Comercio, № 35, BID-INTAL, Washington, pp. 19-36

SCHNEIDER, William (1976), Food, foreign policy, and raw materials cartels, National Strategy Information Center, Nueva York

STRANGE, Susan (1982), "Cave! Hic dragones: a critique of regime analysis", en KRASNER, Stephen (ed.) (1983), International regimes, Cornell University, Ithaca, pp. 337-354
STRANGE, Susan (1994), States and markets, $2^{\text {nd }}$ ed., Continuum, Londres

VADELL, Javier (2012), “El dragón en el patio trasero: China, Sudamérica y el Consenso del Pacífico", en SERBIN, Andrés et al. (coord.), El regionalismo "post-liberal" en América Latina y el Caribe, CRIES, Buenos Aires, pp. 397-434 